\title{
Proceedings of the meeting of the Association of British Neurologists, in Belfast, 3-5 April 1986
}

A SEROLOGICAL TEST FOR THYMOMA IN PATIENTS WITH MYASTHENIA GRAVIS

V Patterson, $\mathbf{R}$ Alderdice, $\mathbf{M}$ Haire. Department of Neurology, Royal Victoria Hospital and Department of Immunology, Queens University, Belfast

Ten to fifteen percent of patients with myasthenia gavis (MG) have an underlying thymoma. Such patients do less well and it may be important to detect them early. Conventional tests for thymoma such as immunofluorescent demonstration of skeletal muscle antibodies and CT scanning of the anterior mediastinum may give false positive and false negative results. We have attempted to validate a report that antibodies to a citric acid extract (CAE) of muscle, demonstrated by an indirect haemagglutination technique, are specific for thymoma. We studied four groups of subjects - normal controls, patients with other neuromuscular disease (OND), MG patients with known thymic histology, and MG patients with unknown thymic histology. CAE antibodies were not detected in any normal controls or patients with OND. In the group with known thymic histology they were found in all those with thymoma and none of those with other histology. They were found in about $40 \%$ of those with unknown thymic histology. Our experience so far suggests that demonstration of CAE antibodies is a specific and sensitive test for thymoma in patients with MG.

RHEUMATOID ARTHRITIS AND MOTOR NEURONE DISEASE - AN ASSOCIATION? RA Metcalfe, W Schady. Department of Neurology, Manchester Royal Infirmary

Three patients with rheumatoid arthritis (RA) and motor neurone disease (MND) are presented. They had had "classic" RA (American Rheumatism Association criteria) for 2, 19 and 23 years respectively before they developed neurological symptoms. Lower limb weakness was the presenting complaint in all patients, followed by footdrop, distal upper and lower limb wasting and bulbar symptoms in two. Tongue fibrillation was evident in these two patients whereas all had a brisk jaw jerk and a combination of upper and lower motor neurone signs in the limbs with intact sensation.
Serum creatine kinase was moderately elevated in one. There were electromyographic signs of widespread chronic partial denervation with abundant spontaneous activity in the presence of normal motor conduction velocities and sensory action potentials. The CSF contents were normal. Quadriceps muscle biopsies revealed angular fibres and small fibre type grouping characteristic of partial denervation. Relentless progression of the disease resulted in the death of two patients after 20 and 34 months respectively, the surviving patient being wheelchairbound 23 months after presentation.

This association raises important issues regarding the aetiology of so-called motor neuropathies in collagen disorders and the possibility of immunological mechanisms in some forms of MND.

CONTROLLED TRIAL OF A TRH ANALOGUE (RX77368) IN PATIENTS WITH MOTOR NEURON DISEASE

RJ Guiloff, C Demain, D Eckland, S Lightman. Department of Neurology and Medical Unit, Westminster Hospital, Charing Cross and Westminster Medical School, London

Following a pilot study on five patients, twenty-six patients entered a double blind trial of intravenous L-pyroglutamyl-L hystidyl-L-3,3 dimethyl prolineamide (RX77368) $300 \mu \mathrm{g} / \mathrm{kg}$ over two hours randomly crossed over with physiological saline after seven days. RX77368 was preferred to placebo by 10 of 13 patients with bulbar syndrome. Temporary improvement in dysarthria in eight patients was documented with phonaemic analysis; four with anarthria did not improve. Other bulbar mediated functions assessed included vital capacity, peak flow, maximal inspiratory and expiratory pressures, swallowing, palatal and tongue movements. Dynamometry measurements showed significant change in coefficient of variation (CV) of maximal isometric voluntary contraction in some muscle groups. Trend analysis showed increase or decrease in force in muscle groups with change in CV. Changes in force were seldom clinically significant. Tone changed in eight patients and fasciculations increased in 18 No detectable change in reflexes, fatigability nor Norris scale were seen. Neurological effects lasted up to 72 hours. There was significant increase in fibre density and median macro EMG amplitude but not in compound muscle action potential of biceps. Thirteen patients had no significant side effects. Serum thyroxine increased and returned to basal levels within seven days.

A TWO CENTRE STUDY OF THE

ELECTRORETINOGRAM (ERG) IN MULTIPLE SCLEROSIS

D Papakostopoulos, F Fotiou, NK Banerji. Burden Neurological Institute, Bristol, England

Thessaloniki University, Greece

To investigate the possibility of retinal involvement in MS 10 patients with positive McAlpine criteria, monocular retrobulbar neuritis and duration of disease between 17 and 5 years, were studied in Thessalonikio and 5 in Bristol.

The ERG to white light flash from light adapted eyes was recorded with infraorbitale skin electrodes using a method previously reported (Papakostopoulos 1982. In GA Chiarenza and D Papakostopoulos (Edsto Clinical Application of Cerebral Evoked $\leqq$ Potentials in Paediatric Medicine. Exerpta Medica, Amsterdam). The PR VEPs from $\mathrm{Oz}$, referred to $\mathrm{Fz}$, were also recorded.

All patients in Thessaloniki had abnormal PR VEP latencies when the abnormal eye was stimulated. The ' $b$ ' wave of the ERG of the abnormal eye $(x=6.4 \mu \mathrm{V}+1.8)$ was consistently smaller $(x=10.5 \mu \mathrm{V} \pm 2.3)$ in amplitude compared with the normal eye (p $<0.001$ ) or 10 normal control subjects' eyes $(x=11.2 \mu V+2.9)(p<0.001)$. The recordings in Bristol yielded similar results.

These results, obtained in two different laboratories, provide neurophysiological evidence of damage additional to demyelination in the central nervous systems in multiple sclerosis.

AN INVESTIGATION OF NEUROLOGICAL, PSYCHOLOGICAL AND PSYCHIATRIC IMPAIRMENT IN MULTIPLE SCLEROSIS R Brown, J Kogeorgos, C Kennard, DF Scott, Department of Neurology and Department of Clinical Neurophysiology, The London Hospital, Whitechapel, London 
The presence of clinically silent lesions in multiple sclerosis (MS) is a well recognised feature of the disease, but raises the question whether resultant signs might remain undetected because of limited testing procedures. This present study attempted to explore such a possibility by under-taking detailed assessment using a range of diverse investigative techniques, including neurological, psychological and psychiatric tests.

In this study, 82 patients with "definite MS" (53 females and 29 males, age range 20-60 years with mean of 42 years) were assessed using the Kurtzke Disability Status Scale, a standard intelligence scale with tests of selected neuropsychological functions, the Beck's Depression Scale and the Goldberg Standardised Interview. They were compared with 42 control patients (21 females and 21 males, age range 20-60 with a mean of 46 years) suffering from chronic disabling non-cerebral neurological conditions.

Results showed both groups to be intellectually below average with no significant differences on estimated premorbid IQ, or neuropsychological functions, where both groups performed below expected levels for their age groups. The MS patients showed a higher degree of psychopathology which consisted predominantly of depressive symptoms, including irritability and lack of concentration, as well as a characteristic feeling of excessive fatigue.

THE CLINICAL AND PATHOLOGICAL FEATURES OF CORTICAL LEWY BODY DISEASE

WR Gibb, AJ Lees. The National Hospitals for Nervous Diseases, London

Severe loss of pigmented substantia nigra cells with the presence of Lewy bodies are the characteristic histological features of L-dopa responsive Parkinson's disease. Small numbers of Lewy bodies may also be found in the hippocampus in about a quarter of cases. A possibly distinct clinicopathological entity termed diffuse Lewy body disease has also recently been described in the literature, with multiple Lewy bodies present in the fifth and sixth layers of the cerebral cortex. The clinicopathological features of four patients with this condition will be reported and a review of the literature given.

\section{CSF INTERFERON IN NEUROLOGICAL} DISORDERS

RJ Abbott, I Bolderson, P Gruer. St James' University Hospital, Leeds

There are three antigenically distinct sub- types of interferon (IFN). Studies using bio- aware of any other similar cases of Influenza assays have suggested that IFN- $\alpha$ and IFN- B encephalitis in the literature.

$\beta$ are produced in response to viral infection, whereas IFN- $\gamma$ production is induced by immunological mechanisms. IFN- $\gamma$ will induce the expression of class II MHC antigens in tissues which do not normally express them. It has been suggested that this mechanism may trigger autoimmunity.

Using highly sensitive and specic immunoradiometric assays (IRMA) we have measured IFN- $\alpha$ and IFN- $\gamma$ in CSF of patients with infectious and immunologically mediated neurological disorders. Both immunoreactive IFN $-\alpha$ and IFN- $\gamma$ were present in the CSF in the acute phase of viral meningitis, but no IFN was found in other neurological disorders such as multiple sclerosis and Guillan-Barré Syndrome.

IRMAs have not been previously applied to the study of IFN in CSF, and INF- $\gamma$ has not previously been detected in CSF. Its presence in CSF following viral infection gives support to the hypothesis that a preceding viral infection may trigger autoimmune disorders which involve the nervous system.

ENCEPHALITIS DUE TO INFLUENZA B VIRUS SA Hawkins, JH Connolly, JA Lyttle. Department of Neurology, Royal Victoria Hospital, Belfast

During an epidemic of Influenza B/Singapore/222/79 in Belfast, two cases of severe encephalitis were seen with diagnostic rises of antibody titres to the specific virus strain during the course of the illness. One was in a 37-year-old woman who had generalised encephalitis with generalised EEG slowing, and lymphocytic pleocytosis in the CSF. She was hospitalised for four months with prolonged stupor, epilepsy and myoclonus. She eventually made a full recovery. The second case was a 38-year-old woman who had a four day prodromal illness, became dysphasic, with associated right arm weakness. Two days later she had a solitary convulsion. CT scan was normal, EEG showed generalised changes in keeping with encephalitis, maximal in the left temporal region. CSF showed a lymphocytic pleocytosis. She made a rapid recovery, was discharged within two weeks and was back to work three months later.

The features of the illness and timing of the rises in antibody titres are more in keeping with encephalitis than post-infectious encephalomyelitis. Other neurological syndromes have in the past been rarely associated with Influenza B infection. We are not
A COMPARATIVE STUDY OF PROGABIDE,
VALPROATE AND PLACEBO AS ADD-ON THERAPY IN PATIENTS WITH REFRACTORY EPILEPSY

P Crawford, D Chadwick, Mersey Regional Department of Neurology, Liverpool

Progabide is a gaba agonist with antiepileptic properties in animal models of seizures and epilepsy. Its efficacy in human epilepsy is less certain.

A three way single blind cross-over comparison of progabide, valproate and placebo, as adjunctive therapy for 6 months each, was undertaken in 64 patients with therapy resistant partial and generalised seizures. The study was not completed because of the incidence of elevated hepatic enzymes on progabide. Analysis of efficacy showed progabide to be inferior to valproate against all seizure types, and particularly against tonic-clonic seizures. Valproate was superior to placebo against all seizure types, partial and tonic-clonic seizures. Progabide did not differ significantly from placebo in any instance. In addition progabide caused elevation of hepatic enzymes which was symptomatic in one case, and was associated with an interaction with phenytoin which results in intoxication in some cases.

\section{PROPOSED RESEARCH DIAGNOSTIC CRITERIA} FOR PARKINSON'S DISEASE

CD Ward, WR Gibb. University Department of Rehabilitation, Southampton General Hospital

In the absence of pathognomonic markers, the diagnosis of Parkinson's disease (PD), and its distinction from other Parkinsonian syndromes, depends on clinical observation. Published descriptions of the motor syndrome of Parkinsonism are often unnecessarily ambiguous. There is a lack of consistency in the use of even the three major terms, "tremor" "rigidity", akinesia". We present the first detailed proposals for research diagnostic criteria for PD, based on review of published clinical series and studies relating clinical features of Parkinsonian syndromes to pathology. Clinical information is assigned to four categories; 1) background (eg genetic); 2) Prodromal and presenting features other than Parkinsonism; 3) The motor syndrome of Parkinsonism; 4) Additional features. Findings are further classified as typical, atypical or incompatible. We have designed a diagnos- 
tic form which facilitates the assessment and description of both individuals and series of patients. The scheme is necessarily tentative but will, we hope, be refined in future discussions.

BILATERAL OCCLUSION OF THE INTERNAL CAROTID ARTERIES: PRESENTING SYMPTOMS IN 74 PATIENTS AND A PROSPECTIVE STUDY OF 34 MEDICALLY TREATED PATIENTS JPH Wade, W Wong, HJM Barnett. University Hospital, London, Ontario

Seventy-four patients out of the total of 1377 subjects entered into the International EC/1C Bypass Study had atherosclerotic occlusion of both internal carotid arteries. All had suffered either ischaemic hemisphere infarcts $(80 \%)$ and/or transient ischaemic attacks $(80 \%)$ involving one $(78 \%)$ or both $(22 \%)$ carotid territories. In addition, 10 subjects $(14 \%)$ incurred recurrent vertebrobasilar or presyncopal episodes, many of which appeared secondary to haemodynamic insufficiency. The prevalence of risk factors and concomitant vascular disease was high; $93 \%$ gave a history of heavy smoking.

Thirty-four subjects were treated conservatively and prospectively followed for a mean period of 42 months. Eighteen patients $(52.9 \%)$ suffered further cerebrovascular events, giving a rate of $15 \cdot 1 \%$ per patient year; these were isolated TIAs in seven subjects and stroke in 11 cases. The stroke rate was $9 \cdot 2 \%$ per patient year. Patients who had presented with ischaemic events involving more than one carotid territory were significantly more prone to subsequent cerebral infarction than those in whom symptoms had been confined to one carotid territory $(p<0.04)$. Deaths per year amounted to $8 \cdot 4 \%$. Seventy-one per cent survived, of whom half were either symptom-free or minimally disabled at the end of follow-up.

In the group of pre-selected patients, which excludes those with severe deficits at presentation, bilateral occlusion of the carotid arteries may be a condition compatible with useful existence.

THE CLINICO-PATHOLOGICAL CORRELATION OF LACUNAR SYNDROMES; THE OXFORDSHIRE COMMUNITY STROKE PROJECT

JM Bamford, PAG Sandercock, CP Warlow. University Department of Neurology, Oxford

Several distinct clinical syndromes have been noted to occur in the presence of restricted areas of infarction within the vascular territory of deep penetrating arteries ("lacunes"). However, several reports have suggested that similar clinical syndromes may occur in the presence of lesions at other sites. Both the original observations on lacunar stroke and subsequent reports have been based on very few cases. We have studied prospectively the clinico-pathological correlation of lacunar syndromes in a community based sample of stroke patients.

Over a three year period, 515 first ever strokes were assessed of whom $108(21 \%)$ were considered to have one of the recognised lacunar syndromes - 49(45\%) pure motor stroke, seven $(6 \%)$ pure sensory stroke, nine $(8 \%)$ ataxic hemiparesis and $43(40 \%)$ sensori-motor stroke. A CT scan or necropsy examination was performed in $95(88 \%)$ cases. In $30(32 \%)$ cases there was a small, deep infarct appropriate to the clinical features, in $59(62 \%)$ cases the CT scan was normal and in three $(3 \%)$ cases the CT scan showed primary intra-cerebral haemorrhage. In two other cases there was both deep and cortical infarction which might have caused the clinical features and in one case there was infarction in the territory of the pericallosal artery. These findings allow the significance of individual case reports to be assessed.

\section{MAGNETIC RESONANCE IMAGING IN} EXPERIMENTAL CEREBRAL OEDEMA

D Barnes, WI McDonald, DN Landon, $P$ Tofts, G Johnson. Institute of Neurology, London

MRI readily detects abnormalities in many CNS diseases. The specificity of abnormal images is unknown. We have, therefore, investigated two different types of cerebral oedema in cats: vasogenic cold oedema, where the expanded extracellular space contains variable amounts of plasma protein, and cytotoxic oedema induced by triethyltin, characterised by the accumulation of protein-free fluid in intramyelinic vacuoles.

NMR images, quantitative changes in relaxation times $T_{1}$ and $T_{2}$, and magnetisation decay characteristics were correlated with ultrastructural features of the lesion.

MRI was sensitive to these lesions but the images lacked specificity. The ratio of changes in $T_{1}$ and $T_{2}$, however, was characteristic. In early vasogenic oedema associated with large amounts of protein, $T_{1}$ and $T_{2}$ increased in approximately the same proportion. As the protein content diminished over a period of days, $T_{2}$ tended to be more elevated than $T_{1}$.

In cytotoxic oedema, the proportional increase in $T_{2}$ was approximately twice that in $T_{1}$. The magnetisation decay characteristics of each lesion showed specific features

The images and the quantitative data together distinguish the two lesions and provide an insight into the nature of underlying pathological processes.

X-LINKED BULBO-SPINAL NEURONOPATHY

D Thrush, J Wilde. Department of Neurology, Freedom Fields Hospital, Plymouth Department of Neuropathology, Frenchay Hospital, Bristol

In 1968, Kennedy and colleagues reported 11 males from two families with a distinct form of bulbar and spinal muscular atrophy which was slowly progressive and inherited as an X-linked recessive. Since then a further 31 patients have been described in five separate reports, 10 being described by Harding and colleagues in 1982. As well as reporting similar findings to Kennedy they found reduced or unrecordable sensory nerve action potentials in seven patients and because of this abnormality they named $\mathrm{X}$-linked recessive bulbo-spinal neue ronopathy.

The clinical features of two brothers an one nephew with $\mathrm{X}$-linked recessive bulbo spinal neuronopathy will be described. The neurophysiological investigations and sura nerve biopsy, previously unreported confirmed that both motor and sensor nerve are affected.

Because of the genetic implications we wish to stress the importance of recognising this disorder as a separate entity which should not be classified with the spinal muscular atrophies.

THE ROLE OF CT SCANNING AND THE EVALUATION OF SPINAL MUSCULAR ATROPHY P Sambrook, WJK Cumming. Departments of Radiology and Neurology, University Hospital of South Manchester

Adolescent and adult onset spinal muscular atrophy (SMA) may present as a limb girdle, Fascio-scapulc-humeral or Scapuloperoneal syndrome. Histopathological verification of the diagnosis is obtained from muscle biopsy but this only provides information on the abnormalities in the biopsied muscle. Clinical examination provides information on muscle groups, for example, posterior lower limb muscles, but does not permit differentiation of the relative contribution to muscle strength from individual muscles. 
The development of modern generations of CT scanners has permitted more detailed assessment of the extent of the disease process.

CT scanning has been applied to 75 patients with histologically verified SMA of varied presentation and disease duration. CT evaluation was carried out without knowledge of the clinical features or disease duration.

This study has shown there appears to be a recognisable CT appearance in SMA. This was seen irrespective of the clinical mode of presentation. Abnormalities have been demonstrated in mildly affected individuals within families whose older members have shown marked clinical and CT abnormalities, suggesting that the degree of CT abnormalities depends on the disease duration.

Further studies of CT and SMA may obviate the need for muscle biopsy of more than one family member.

OBLIQUE NEEDLE INSERTION DOES NOT PREVENT POST-LUMBAR PUNCTURE HEADACHE J Morrow, A McAuley, V Patterson. Department of Neurology, Royal Victoria Hospital, Belfast

A characteristic headache, worse on standing and eased by lying, is the most troublesome complication of lumbar puncture (LP), occurring in about $30 \%$ of patients in most series. Use of a small-sized needle has been shown to reduce the incidence of post-lumbar-puncture headache (PLPH) and there are anecdotal reports that oblique insertion of the needle does so also. To test this suggestion patients undergoing diagnostic LP were randomly assigned to direct or oblique needle insertion, the other features of the procedure being the same in all subjects. The incidence of PLPH and other complications was determined one and 14 days after the LP by an observer who was unaware of the technique used. At day 1 PLPH was present in 10 of 42 patients who had a direct LP but only in 4 out of 31 whose LP was oblique. However, at 14 days there were 13 headaches in the direct group but now there were 9 in the oblique group. Thus under the conditions used oblique LP does not significantly reduce the incidence of PLPH. It is important in trials on PLPH to extend follow-up beyond the first day.
SERIAL QUANTITATIVE ELECTROMYOGRAPHY (EMG) AND MAXIMUM QUADRICEPS STRENGTH IN PERIPHERAL NEUROMUSCULAR DISORDERS DJ Short, CM Wiles. Department of Neurology, St Thomas' Hospital, London

A comparison has been made between serial measurement of mean potential duration (MPD) and knee extension force in patients with peripheral neuromuscular disorders.

The maximum voluntary isometric knee extension force (MVC) was measured with the subject seated in a special chair. EMG was recorded from vastus medialis using a concentric needle electrode through 2 skin insertions between 5 and $10 \mathrm{~cm}$ proximal to the upper border of the patella. Action potentials from at least 20 individual motor units recruited at low force were recorded on photosensitive paper and subsequently analysed manually to give the mean potential duration (MPD). In addition patients completed a visual analogue scale of their subjective impression and a functional assessment made. A normal range for MPD was established in 25 subjects (aged 28-65). In 10 subjects the coefficient of variation for paired measurements was $3 \%$.

Satisfactory studies were obtained on at least 3 occasions from 20 patients with various myopathies and diabetic amyotrophy.

MPD and MVC changed in the expected direction during clinical improvement or deterioration but in only a few patients was there close correlation. Changes in MPD were a less sensitive quantitative guide to clinical status than maximum voluntary knee extensor force.

\section{THE EFFECTS OF TRH ON PRIMARY LATERAL SCLEROSIS}

P Pinelli, A Villani, F Pisano. Medical Center, Neurological Department, Veruno (NO) Italy

Two patients of the same family (CM, f, 56 years old; AG, m, 35 years old) affected by Strümpell-Lorain disease and one (CR, m, 30 years old) affected by primary lateral sclerosis since 11 years were evaluated by means of clinical, dynamometric, and electromyographic investigations (quadriceps, biceps femoris, tibialis ant. and triceps surae): motor unit voluntary maximal recruitment ( $\mathrm{Vmx}$ ), $\mathrm{Mmx}$ response, $\mathrm{T}$ reflex and $H$ response were recorded with macroelectrodes, and the Wartenberg test was evaluated with simultaneous goniometric recording.

The investigations were repeated thrice before and $20^{\prime}-45^{\prime}$ after each TRH-T admin- istration ( 2 and $4 \mathrm{mg} / \mathrm{im}$ ) and after placebo.

Significant improvement was observed in comparison with placebo (P) $45^{\prime}$ after $2 \mathrm{mg} / \mathrm{TRH}$ (II) and particularly after $4 \mathrm{mg} / \mathrm{TRH}$ (IV) in the patient CR whose spasticity was the most severe. Vmx: $\mathrm{P}+$ $8 \%$; II $+25 \%$; IV $+45 \%$.

First Wartenberg oscillation: $\mathbf{P}+15 \%$; II + $20 \%$; IV + $27 \%$.

Phasic stretch reflex: $\mathrm{P}-25 \%$; II $-55 \%$; IV $-50 \%$.

Improvement in gait veolocity: $\mathrm{P}+20 \%$; II $+36 \%$; IV $+42 \%$.

Some improvement (IV) was still evident $24 \mathrm{~h}$ later.

\section{SHOULDER ABDUCTION FATIGUE}

J Nicklin, D Short, M Wiles. National Hospitals for Nervous Diseases, London

Shoulder abduction fatigue has been assessed in normal subjects and patients with peripheral neuromuscular diseases. Maximum voluntary isometric strength was measured in the non-dominant shoulder abductors, with the subject seated, in ten brief successive contractions over a period of 50-55 seconds using a hand held myometer. A fatigue index (FI) was calculated as $\varrho$ the difference between the means of the first $\stackrel{D}{\circ}$ and last two contractions expressed as a percentage of the first two. Maximum force was not critically dependent on shoulder angle.

In twenty-five normal males (aged 20-60, median 34 years) force declined from the first to the fifth contraction and then remained constant: $\mathrm{F} 1$ was $6 \cdot 1+/-6.7 \%$ (mean $+/-$ sd). In twenty-five normal females (aged 20-65, median 30 years) force increased from the first to the second contraction and then declined: FI was $6 \cdot 1+1-$ $6.4 \%$, no different to males. FI was independent of initial strength and age in this limited sample.

Most patients with myasthenic disorders, on treatment, showed excess fatigue unrelated to weakness as did many with mitochondrial myopathy. Excess fatigue rarely occurred in other disorders. The technique provides a convenient method of documenting strength and fatiguability in this muscle group.

\section{GABAPENTIN IN PATIENTS WITH EPILEPSY P Crawford, D Chadwick. Mersey Regional} Department of Neurology, Liverpool

Gabapentin is a gaba analogue with antiepileptic properties in animals. A double blind, cross-over, add-on trial with 3 
differing doses ( $300 \mathrm{mg}, 600 \mathrm{mg}, 900 \mathrm{mg} /$ day) for 2 months each, was undertaken in 25 patients with resistant partial and generalised epilepsies.

There was a dose-related reduction in seizure frequency compared with a baseline period. ( $\mathrm{P}>0.0001$, Wilcoxon signed rank test). At the highest dose, $43 \%$ of patients showed a greater than $50 \%$ reduction.

No serious adverse effects or drug interactions were noted. The compound appears worthy of further evaluation.

NORMAL CELLULAR SENSITIVITY TO X-RAYS IN PARKINSON'S DISEASE AND ALZHEIMER'S DISEASE

PD Lewis, N Kumar, SA Sabovljev. Royal Postgraduate Medical School, London

It has been claimed that cells from patients with one of several sporadic neurological disorders, including Parkinson's disease and Alzheimer's disease, are hypersensitive to $X$ rays, implying deficiency of DNA reparative processes. We have been unable to confirm increased cellular radiosensitivity in these two conditions. Forearm skin biopsies from three patients with Parkinson's disease (PD) and four with Alzheimer' disease (AD) were cultured and survival of fibroblasts following exposure to graded doses of $\mathrm{X}$-rays was measured by counting cell colonies and assessing the fraction surviving. PD cells (patients aged 41-76) showed a mean $D_{0}$ of 1.21 Gy (12 experiments). AD cells (patients aged 48-62) showed a mean $D_{0}$ of $1.24 \mathrm{~Gy}$ (11 experiments). Control cells grown in parallel showed a mean $D_{0}$ of 1.29 Gy $(22$ experiments). These findings do not give support to the idea that DNA repair deficiency is a major pathogenetic mechanism in degenerative neurological disease.

IMMUNOELECTRON MICROSCOPICAL EVIDENCE OF DUAL HORMONE PRODUCTION IN PITUITARY ADENOMA CELLS

CHS Cameron, CO Ozo, IV Allen, L Kennedy, AB Atkinson, DR Hadden. Departments of Pathology, Neuropathology and Endocrinology, Royal Victoria Hospital, Belfast

A pituitary adenoma was surgically removed from a 42-year-old male presenting with classical symptoms of acromegaly plus decreased libido and impotence. The lowest serum growth hormone $(\mathrm{GH})$ level during oral glucose tolerance test was $26.5 \mathrm{mu} / 1$. Prolactin (PRL) levels ranged from $1990-4790 \mathrm{mu} / 1$

Histologically the adenoma was predominantly acidophilic. With immunoperoxidase, a significant number of cells stained positively for PRL, while only occasional scattered cells show reactivity for $\mathrm{GH}$. Staining for all other hormones was negative. The tumour had a uniform ultrastructural appearance. The cells showed good cytoplasmic differentiation in keeping with a prolactinoma. Immunoelectronmicroscopy, including double labelling techniques using selected colloidal gold particles as markers, not only demonstrated PRL and GH in each of the tumour cells, but also within the same granules.

The results indicate the presence of a pituitary adenoma in which the cells are capable of simultaneously producing $\mathrm{GH}$ and PL and packaging them within the same secretory granule. This is thought to repre- sent a mammosomatotroph cell adenoma. Although such a mechanism for the production of two or more hormones by a single tumour cell has previously been postulated, we believe our report is the first time this has been shown by this technique.

\section{CLINICAL, HISTOLOGICAL AND}

ULTRASTRUCTURAL FINDINGS IN A FAMILIAL MUSCLE DISEASE WITH TUBULAR AGGREGATES S Cameron, V Patterson, I Allen. Department of Neuropathology, Queens University and Department of Neurology, Roya Victoria Hospital, Belfast

Tubular aggregates (TAs) are an unusual histological finding in muscle disease. They most often occur in type 2 fibres in patients with aches, cramps and pains but may also be found in small numbers in a variety of other conditions. Two families with muscle disease in which TAs were the dominant biopsy feature have recently been described and we report a third family of what seems to be a distinct condition. A father and daughter had symptoms of arm and lego weakness starting in their early teens witlo very slow progression. Both had proxima weakness with some limitation of eye move $\frac{}{\mathbb{C}}$ ments and Achilles tendon contractures? Creatine kinase ws 5-10 times normal. His tology and histochemistry of muscle biop sies showed vacuoles in almost all fibres and electron microscopy revealed that these were filled with TAs. These consisted of cloself packed parallel tubules and differed from the TAs most commonly reported in other conditions. The pathogenesis of these remarkable structures is not clear. 